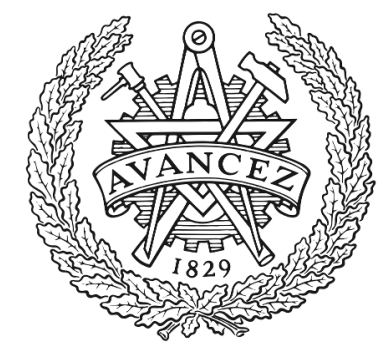

\title{
CHALMERS
}

UNIVERSITY OF TECHNOLOGY

\section{A Congestion Forecast Framework for Distribution Systems with High Penetration of PV and PEVs}

Downloaded from: https://research.chalmers.se, 2023-04-26 10:56 UTC

Citation for the original published paper (version of record):

Srivastava, A., Steen, D., Le, A. et al (2019). A Congestion Forecast Framework for Distribution Systems with High Penetration of PV and PEVs. 2019 IEEE Milan PowerTech, PowerTech 2019. http://dx.doi.org/10.1109/PTC.2019.8810871

N.B. When citing this work, cite the original published paper. 


\title{
A Congestion Forecast Framework for Distribution Systems with High Penetration of PVs and PEVs
}

\author{
Ankur Srivastava, David Steen, Le Anh Tuan, Ola Carlson \\ Division of Electric Power Engineering \\ Department of Electrical Engineering \\ Chalmers University of Technology \\ 41296 Gothenburg, Sweden \\ Email: \{ankur.srivastava, david.steen, tuan.le, ola.carlson\}@chalmers.se
}

\begin{abstract}
This paper presents a congestion forecast framework based on probabilistic power flow for electrical distribution systems with high penetration of solar photovoltaic and plugin electric vehicles. The proposed framework is tested using data of the real distribution grid of Chalmers campus for case studies to analyze the impact of different local production levels and operating modes of solar photovoltaic inverters. The results have shown that cumulative probability for network congestion in branches and transformers would increase by $30 \%$ and $20 \%$, respectively, in the case when level of local solar photovoltaic production, demand, and number of plug-in electric vehicles is increased by $100 \%, 95 \%$, and $100 \%$, respectively. It has also been shown that network congestion in branches and transformers is $4 \%$ and $8 \%$, respectively, more likely to occur in constant-V mode as compared to constant-pf mode. This framework could be used by distribution system operators to predict network congestion.
\end{abstract}

Index Terms-Backward-forward sweep, congestion forecast, electric vehicles, photovoltaics, probabilistic power flow

\section{INTRODUCTION}

With the growing concern towards global warming, the universal focus has been on the transition towards low-carbon energy systems. Two major technological shifts in this regard are replacement of conventional coal-based power generation by renewable energy resources and hydrocarbon-fuelled vehicles by electric vehicles (EVs). The International Energy Agency presented in their annual report of photovoltaic power systems programme 2017 that solar photovoltaics (PVs) is now the fastest growing energy technology [1]. Both PVs installation and EVs sales have increasing growth rates worldwide, raising their share's to new heights every year.

Increased penetration of PVs and EVs in distribution systems brings various environmental and economic benefits, but their associated intermittency also brings challenges for distribution system operators (DSOs), including e.g., voltage fluctuations, distribution network congestion [2]. Due to solar irradiance and temperature uncertainties associated with PVs and varying charging demand associated with EVs, deterministic power flow methods are unable to incorporate them in power flow model. Thus, generally, a probabilistic approach is adopted to account for these uncertainties. Probabilistic

The work leading to this paper was funded through the UNITED-GRID project which has received funding from the European Community's Horizon 2020 Framework Programme under grant agreement no. 773717. approach incorporates the uncertainties associated with input variables through their probability distribution function (PDF).

Probabilistic approach has been very useful in uncertainties modelling. For PVs uncertainties modelling, the authors in [3] developed a probabilistic load model to be included in probabilistic power flow (PPF), a probabilistic algorithm for power reserve evaluation with high PVs penetration is developed in [4], a chronological probabilistic model is developed in [5], a polynomial chaos expansion method for inclusion of nonGaussian random variables and polynomial nonlinearities in [6]. Similarly, for modelling of EVs uncertainties, a probabilistic approach along with queuing theory is used in [7], correlation among inputs are considered using probabilistic approach in [8], a stratified latin hypercube sampling based probabilistic approach is used in [9]. Some papers as presented in [10] and [11] have simultaneously considered the uncertainties associated with PVs and EVs. These research works have not dealt with the development of such visualization tools which can be directly used by DSOs for analysis of their network operating conditions and then help them in taking suitable action. A computationally efficient tool for DSOs is proposed in [12] to assess the impact of uncertainties associated with distributed units. Also, another probabilistic method has been proposed in [13] to detect and rank the congested lines for the grid operators. However, a well developed visualization framework for network operating conditions is required for DSOs. Also, in context of operating requirements for PVs inverters, analysis of the impact of different operating modes could be very interesting from the DSOs perspective.

This paper develops a congestion forecast framework based on PPF considering uncertainties associated with PVs, plugin electric vehicles (PEVs) and other conventional loads. The framework also considers the different operating modes of PVs inverters, which in-turn reflect the requirements for connecting PVs to distribution systems. The most important function of the proposed congestion forecast framework is the visualization of the network congestion based on cumulative probability of the proposed congestion indicators, including node voltage deviation, branch and transformer overload, for the distribution system and its components. These indicators can help DSOs in analysing congestion in their network and then take appropriate measures to manage network congestion. 
The rest of the paper is organised as follows, Section II presents the modelling of the distribution system and components. Section III explains the proposed congestion forecast framework. Section IV discusses the case studies and results. Section V outlines the concluding remarks.

\section{Modelling OF Distribution System AND COMPONENTS}

This section introduces the different components considered in the distribution systems and their modelling.

\section{A. PVs and Load Forecast}

With increased penetration of PVs in the distribution systems, the intermittency associated with the generation also increases. The electrical loads in the distribution systems are variable, especially with emergence of new types of loads such as EVs, heat-pumps, etc. In order to effectively manage and control distribution systems, inclusion of PVs and load forecast could play an important role. There are well-established PVs and load forecast methods depending upon desired forecast horizon which could vary from a few minutes to days [14]. For simplicity, this work considers PVs and load forecast to be available as inputs to proposed congestion forecast framework.

\section{B. Operating Modes of PVs-Inverter}

Earlier, PVs-interconnection with the grid had limited controls. For instance, IEEE DERs-interconnection standard 2003 [15] states that all inverters are supposed to be operated at unity power factor in grid-connection mode. But now, when local renewables production (especially PVs) is increasing, several changes are proposed in the DERs-interconnection standards. There are different performance requirements presented in recent IEEE DERs-interconnection standards report [16].

The PVs-inverter control characteristic can be expressed as:

$$
P_{i n j}{ }^{2}+Q_{i n j}{ }^{2} \leq S_{\text {rated }}{ }^{2}
$$

where, $S_{\text {rated }}$ represents the rated capacity of PVs-inverter, $P_{i n j}$ and $Q_{i n j}$ represents active and reactive power injections.

With scenarios where level of $P_{i n j}$ is high, it might lead to severe node voltage fluctuations, and then inverter control switches $Q_{i n j}$ from over-excited to under-excited mode to bring voltage within limits. Further, for cases, where $P_{i n j}$ and corresponding $Q_{i n j}$ does not satisfy (1), then inverter control is forced to curtail $P_{i n j}$, so that constraint is satisfied and node voltage are brought within limits. This work considers the following two modes of operation to mimic different inverter controls:

1) Constant Power Factor Mode: (constant-pf): In this mode, a constant power factor is taken for all the nodes (including generator nodes) and all are modelled as $P-Q$ nodes. The generation at generator nodes is modelled as negative load.

2) Voltage-Reactive Power Mode: (constant-V): In this mode, generator nodes are modelled as $P-V$ nodes i.e., for a range of $P_{i n j}$, voltage remains fixed with support through reactive power $Q_{i n j}$ from inverter, such that (1) is satisfied.

\section{PEVs Charging Power Demand Model}

The transition towards future distribution systems with increasing share of PEVs, motivates for their impact analysis on electrical systems. In some urban areas, the overall PEVs charging demand could be high and possibly coincide with system peak demand, leading further to system congestion, voltage fluctuations, etc. Thus, the overall charging demand at a PEVs charging station should be modelled accurately as it depends on several factors such as number of vehicles, vehicle charging time, and vehicle charge status.

The model presented in [7] has been modified and used in this work for the detailed modelling of total power demand at a PEVs charging station. Some of the used model parameters are chosen according to the taken test case. In Sweden, around 50000 PEVs have been registered by December 2017. The estimated market share parameters for different class of PEVs in Sweden are presented in Table I [17].

TABLE I

PEVs Class Market Share in Sweden

\begin{tabular}{|c|c|c|c|c|}
\hline & SUV & Mid-size & Economy & Micro \\
\hline$\%$ share & 30 & 40 & 20 & 10 \\
\hline
\end{tabular}

\section{COngestion Forecast Framework}

This section explains the congestion forecast framework along with the details of the probabilistic power flow model used in this work. The functional diagram for congestion forecast framework is shown in Fig. 1. The uncertainties associated with PVs, conventional load and PEVs charging power demand are considered in the proposed framework. The probabilistic approach is employed through Monte-Carlo simulations (MCS), each of which leads to a power flow solution. With the obtained power flow solutions, congestion forecast is evaluated through indices such as nodes voltage deviation, branches and transformers overloading.

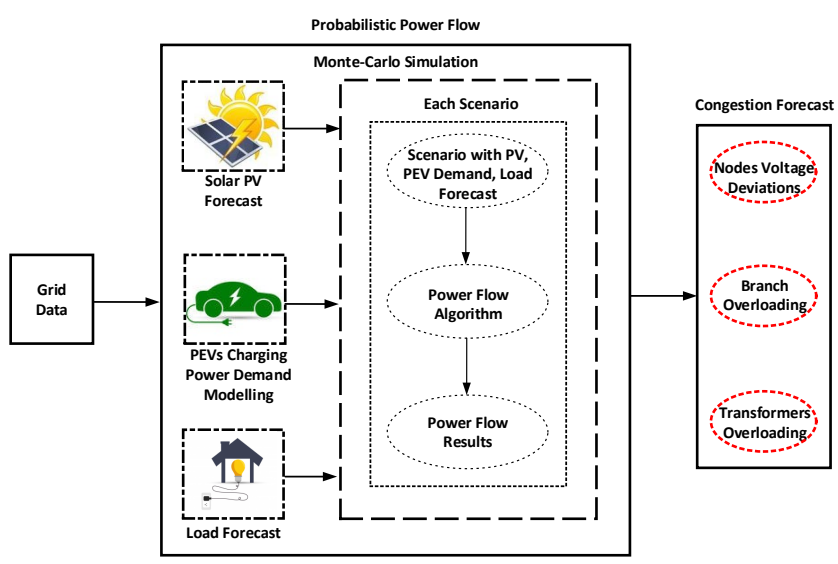

Fig. 1. Functional diagram for the congestion forecast framework

\section{A. Probabilistic Power Flow Method}

The proposed framework uses Monte-Carlo simulations (MCS) for PPF where a large number of scenarios are simulated based on PDF of different variables (uncertainties). This 
work employs backward-forward sweep method for the power flow simulations.

\section{Backward-Forward Sweep Method (BFS)}

BFS method is suitable for highly radial distribution networks whose solution is obtained by iterative solution from two sets of recursive equations. The detailed procedures of BFS method without and with the consideration of $P-V$ nodes in the system, are as follows [18]:

1) Without P-V nodes: Here, all the nodes except the slack node are modelled as $P-Q$ nodes. To start, the voltage at the slack node is kept constant and a flat voltage start is assumed at all other nodes and then for $i^{\text {th }}$ iteration, the following iterative steps are performed:

(a) Calculate current injection at node $k$, as:

$$
C_{k}^{(i)}=\left[\frac{S_{k}}{V_{k}^{(i-1)}}\right]^{*}-Y_{k} V_{k}^{(i-1)} \forall k=1,2, \ldots, n
$$

where, $V_{k}{ }^{(i-1)}$ is the voltage at node $k$ in $(i-1)^{\text {th }}$ iteration, while $S_{k}$ and $Y_{k}$ are the apparent power injection and sum of shunt elements admittances at node $k$.

(b) Calculate branch currents starting from the branch connected to the last node and then moving backward. Thus, the current in branch $j$, is calculated as:

$$
I_{j}{ }^{(i)}=C_{N}{ }^{(i)}+\sum_{f=1}^{T} I_{f}^{(i)} \quad \forall j=b r, \ldots, 2,1
$$

where, $C_{N}{ }^{(i)}$ is the load current at the node $N$ and $T$ is the total number of branches coming out from node $N$.

(c) Update the node voltages starting from the slack node and moving forward towards the last node. The voltage at node $N$ is calculated as follows:

$$
V_{N}{ }^{(i)}=V_{M}{ }^{(i)}-Z_{j} I_{j}{ }^{(i)} \quad \forall j=1,2, \ldots, b r
$$

where, $Z_{j}$ represents the impedance of a branch $j$.

These steps are performed iteratively until the convergence criteria is satisfied.

2) With P-V nodes: Here, all the generator nodes are modelled as $P-V$ nodes while the load nodes are modelled as $P-Q$ nodes. Let us suppose that there are $q P-V$ nodes in the network, then for $\alpha^{\text {th }}$ iteration, the procedure for correction of voltage magnitude is as follows:

(a) Calculate voltage magnitude mismatch for all $P-V$ nodes:

$$
\Delta V_{k}^{(\alpha)}=\left|V_{k}^{(s p)}\right|-\left|V_{k}^{(\alpha)}\right| \quad \forall k=1,2, \ldots, q
$$

where, $V_{k}(s p)$ is the specified voltage value at node $k$.

(b) Calculate the reactive current injection as follows:

$$
\left[I_{Q}\right]^{(\alpha)}=[Z]^{-1}[\Delta V]^{(\alpha)}
$$

where, $Z$ is the real and constant impedance matrix with size as number of $P-V$ nodes. Thus, injected reactive current at $k^{\text {th }}$ node can be calculated as:

$$
I_{k Q}{ }^{(\alpha)}=j\left|I_{k Q}{ }^{(\alpha)}\right|
$$

(c) Calculate the total reactive power requirement $Q_{k R}$, for all $P-V$ nodes, as follows:

$$
\begin{gathered}
Q_{k R}{ }^{(\alpha)}=Q_{k}^{(\alpha)}+Q_{k L} \\
Q_{k}^{(\alpha)}=\operatorname{Im}\left[V_{k} I_{k Q}^{\prime}{ }^{*}\right]^{(\alpha)} \quad \forall k=1,2, \ldots, n
\end{gathered}
$$

where, $Q_{k L}$ is the reactive power load at node $k$ and $I_{k Q}^{\prime}$ is the sum of the required reactive current injection and the load current injection $=\left(I_{k Q}{ }^{(\alpha)}+I_{k L}\right)$.

(d) Check for all nodes whether calculated $Q_{k R}\left(=Q_{i n j}\right)$ corresponding to $P_{i n j}$, satisfies the constraint given by (1). If not, then a new value of $P_{i n j}$ and the corresponding value of $Q_{i n j}$ should be calculated. To do this more effectively, a curve-fitting method is used to express $Q_{i n j}$ as a function of $P_{i n j}$ (for a fixed system topology). Curvefitting is a method for expressing a mathematical function which best fit to a series of data points. Here, $P_{i n j}$ and $Q_{i n j}$ are the data points. This method helps in avoiding the iterative process to find the new value of $Q_{i n j}$.

These steps are performed iteratively until the voltage mismatches for all $P-V$ nodes reaches below the tolerance limit.

\section{B. Congestion Forecast}

MCS are simulated to obtain power flow solution using the above procedure. For simplicity, Gaussian PDF is used for generating samples of generation and load forecast to be used in MCS, as follows:

$$
P D F=\frac{1}{\sqrt{2 \pi \sigma^{2}}} e^{-\frac{(x-\mu)^{2}}{2 \sigma^{2}}}
$$

where, $\mu$ and $\sigma$ represents the mean and standard deviation. In this work, $\mu$ is taken as the forecast value of PVs and load at one time instant, while $\sigma$ is taken as 0.1 for PVs and load forecast for simulation cases.

The PEVs charging power demand samples are generated based on the obtained histogram (tends to follow Weibull PDF) from the modelling done in Section II-C. Finally with the results obtained from power flow solutions, the following indices are proposed to evaluate the congestion forecast:

1) Nodes Voltage Deviation: It is the deviation of node voltage from the specified values. Critical nodes are the ones where deviation is more than a pre-specified value in at least an identified number of MCS simulations. For instance, if deviation is more than $3 \%$ in at least $20 \%$ of MCS simulations, it is considered as a critical node.

2) Branch Overloading: It is the measure of amount of the current flowing in the branches above their rated thermal capacity $\left(I_{c}\right)$. Critical branches are the ones where current flow is more than a pre-specified percentage in at least an identified number of MCS simulations.

3) Transformer Overloading: It is the measure of amount of the MVA power flow in the transformers above their rated MVA capacity $\left(T_{c}\right)$. Critical transformers are the ones where MVA power flow is more than a pre-specified percentage in at least an identified number of MCS simulations. 
The congestion forecast algorithm is given as follows:

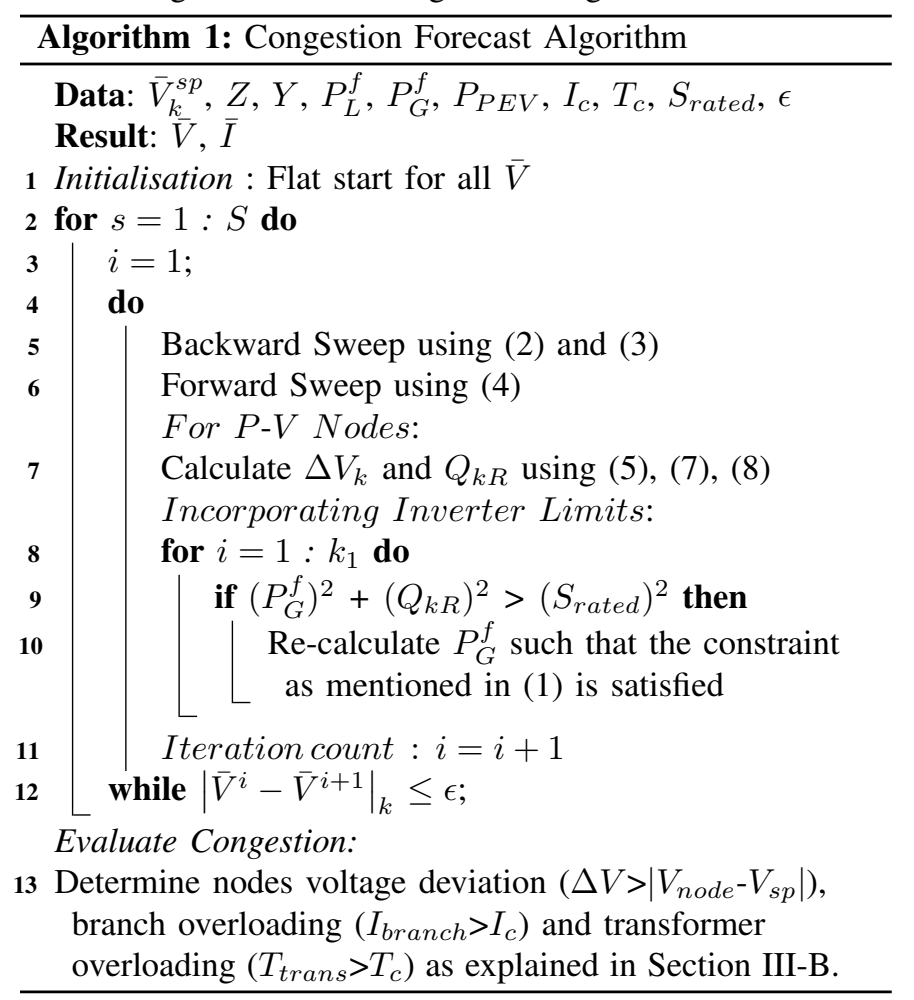

where, $P_{L}^{f}$ and $P_{G}^{f}$ are forecast value of load and PVs, $P_{P E V}$ is estimated PEVs charging power demand, $S$ is number of MCS and $\epsilon$ is tolerance limit.

\section{CAse Studies And Results}

\section{A. Chalmers Campus's Electrical Distribution System}

The proposed congestion forecast framework is applied using the data of modified electrical distribution system of Chalmers University of Technology campus in Sweden. The system is radial and has 40 nodes including medium voltage (MV) and low voltage (LV), 22 branches and 17 MV-LV transformers $(10.5 / 0.4 \mathrm{kV})$. The single-line diagram of Chalmers distribution system is shown in Fig. 2 [19]. The typical system demand varies between 3.5 and $5.5 \mathrm{MW}$. The PVs installed capacity within the system is expected to reach $800 \mathrm{~kW}$ by spring 2019. Also, within the scope of FED project [20], studies have been conducted to estimate the maximum generation capacity through PVs installations. With consideration of both roof-tops and wall-mounted installations it is estimated to be around 9-10 $M W$. The system presently has around 35 PEVs charging points at $32 \mathrm{~A} / 22 \mathrm{~kW}$ and $16 \mathrm{~A} / 3.7 \mathrm{~kW}$ level, located at two charging stations (nodes 7 and 19). The other two charging stations are considered for future scenarios.

The location of PVs injection nodes and PEVs charging stations are shown in Fig. 2, while real recorded Chalmers load data is considered. The rated capacity of PVs-inverter $\left(S_{\text {rated }}\right)$ is taken as $120 \%$ of the maximum PVs capacity, to be considered as defined in (1). Thus, the following two future scenarios are considered as shown in Table II, where PVs generation and load demand are the actual generation and demand for the network at that time instant.



Fig. 2. Single-line diagram of Chalmers $10.5 \mathrm{kV}$ electrical distribution grid

TABLE II

Future Scenarios For Chalmers CAMpus Case Study

\begin{tabular}{|c|c|c|}
\hline Scenario & A & B \\
\hline PVs Generation (MW) & 4 & 8 \\
\hline PEVs Vehicles & 150 & 300 \\
\hline Load Demand (MW) & 4.5 & 8.8 \\
\hline
\end{tabular}

\section{B. Results}

The proposed framework has been implemented in MATLAB (R2016b version). The analysis presents the congestion indicators for one snap-shot while the number of MCS simulations are taken as 10000 and the convergence criteria $\epsilon$ is taken as 0.0001 . The cumulative probability (CP) indicator is chosen to present the results for the congestion forecast (as explained in Section III-B). CP refers to the probability of a variable being greater than or equal to a specific level. For instance, $\mathrm{CP}$ of all branches current flow to be greater than $100 \%$ in scenario B (Fig. 3(b)) is approximately 0.4.

The criteria used for evaluation of congestion indices are presented in Table III. For instance, criteria in case of local production level is that all the nodes where the voltage is more than 1.03 p.u. (or the deviation is more than $3 \%$ ) in at least $20 \%$ of MCS simulations are identified as critical nodes or the branch where current is more than $100 \%$ of rated capacity in at least $20 \%$ of MCS simulations are identified as critical branches. The selection of criteria for evaluation of congestion indices could be adjusted according to the DSOs monitoring requirements and the proposed framework can easily adapt to these requirements.

TABLE III

CRITERIA FOR EVALUATION OF CONGESTION INDICES

\begin{tabular}{|c|c|c|}
\hline Case & $\begin{array}{c}\text { Local Production } \\
\text { Level } \\
\text { (For A and B) }\end{array}$ & $\begin{array}{c}\text { Operating Mode } \\
\text { (constant-pf and -V) }\end{array}$ \\
\hline Critical Nodes & 1.03 p.u., 20\% & - \\
\hline Critical Branches & \multicolumn{2}{|c|}{$100 \%, 20 \%$} \\
\hline Critical Transformers & \multicolumn{2}{|c|}{$100 \%, 20 \%$} \\
\hline
\end{tabular}


The two subsections will present the impact analysis of different local production levels and operating modes as:

1) Impact of Local Production Level: To assess the impact of local production level, two different scenarios A and B as mentioned in Table II are taken with constant-pf mode of operation. The same congestion indices evaluation criteria is used for visualizing the impact of local production level in two scenarios. It can be seen from Fig 3(a) that CP for having a voltage greater than 1.03 at all nodes has increased and similar results can be observed for the critical nodes. From Fig 3(b), the CP for branch currents to be more than $100 \%$ has increased approximately from 0.10 to 0.40 . Also, from Fig 3(c), the CP for transformer MVA loading to be more than $100 \%$ has increased approximately from 0.10 to 0.30 .

Thus, it is evident from the results that with increment of $100 \%$ in local generations, $100 \%$ in number of PEVs vehicles,

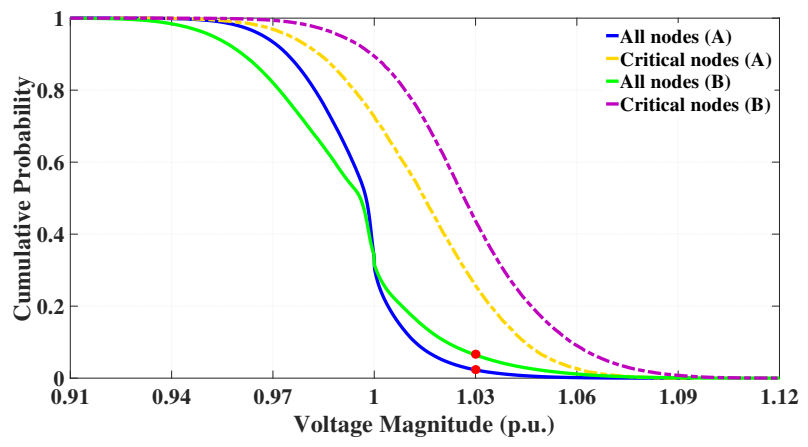

(a) CP for nodes voltage deviation

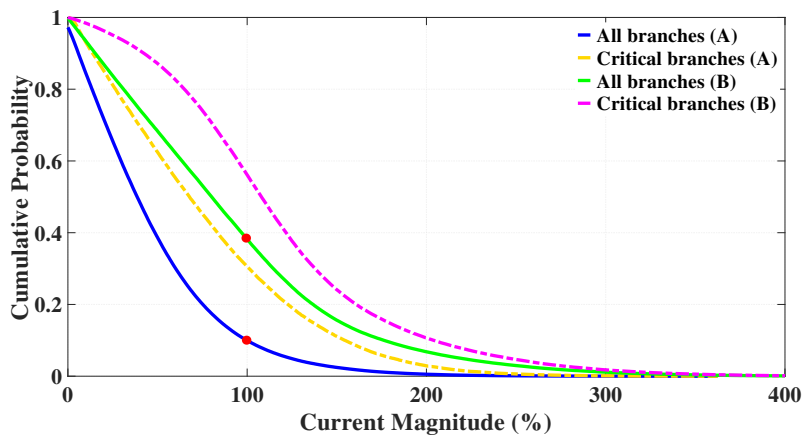

(b) CP for branches overloading

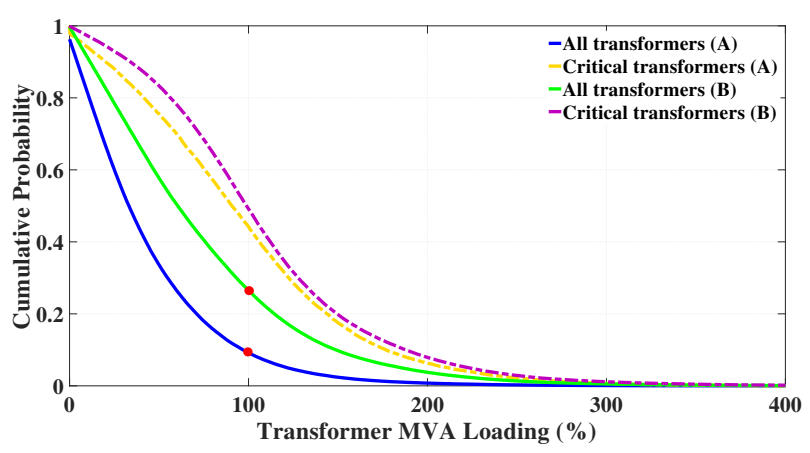

(c) CP for transformers overloading

Fig. 3. Congestion forecast indicators with constant-pf for scenario $\mathrm{A}$ and $\mathrm{B}$ and $95 \%$ in load demand (scenario $\mathrm{A}$ to $\mathrm{B}$ ), the $\mathrm{CP}$ for congestion indices nodes voltage deviation has increased from $2 \%$ to $10 \%$, branch overloading from $10 \%$ to $40 \%$, transformer overloading from $10 \%$ to $30 \%$. These increments are mainly because of higher current, active and reactive power flows, due to higher local production and demand. The nodes are subjected to more fluctuations which lead to higher CP for nodes voltage deviation, and also the $\mathrm{CP}$ for branch and transformer loadings have increased due to higher active and reactive power flow across them.

2) Impact of Operating Modes: To assess the impact of operating modes, scenario B as mentioned in Table II, is simulated under constant-pf and constant- $\mathrm{V}$ modes of operation. The evaluation criteria used for congestion indices is presented in Table III. It can be seen from Fig 4(a) that CP for having a voltage greater than 1.03 at all nodes is almost 0 which

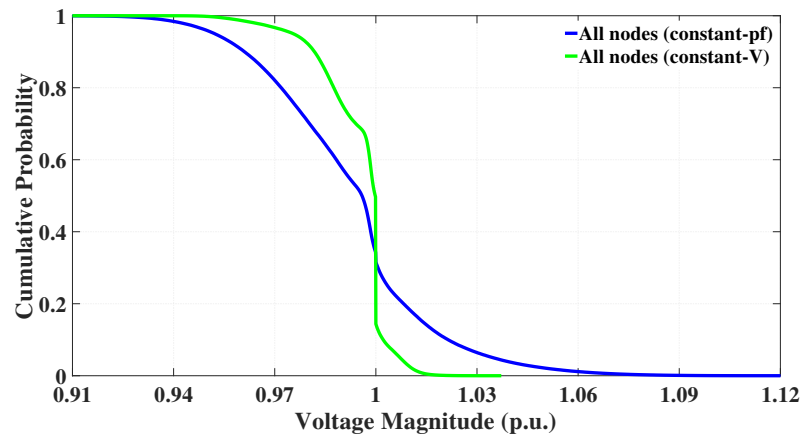

(a) $\mathrm{CP}$ for nodes voltage deviation

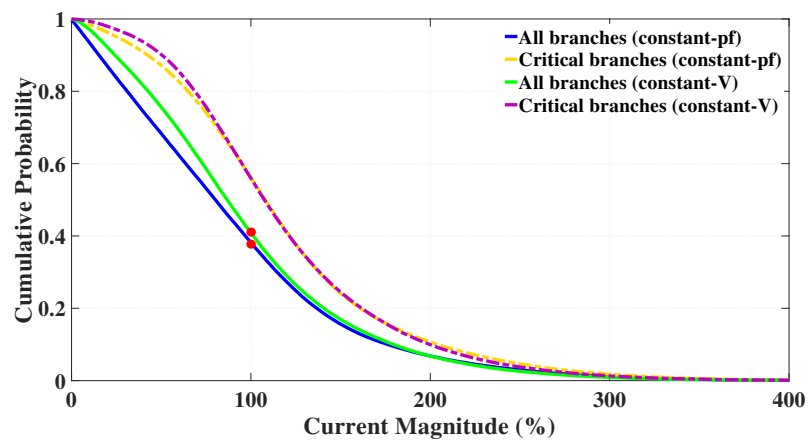

(b) CP for branches overloading

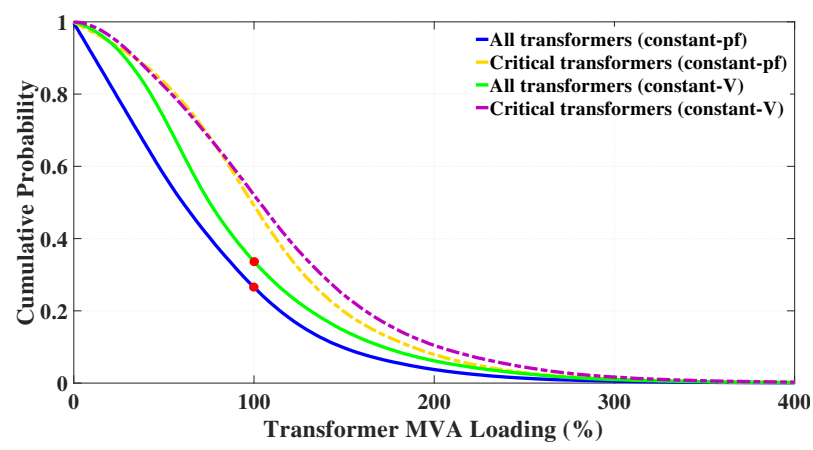

(c) $\mathrm{CP}$ for transformers overloading

Fig. 4. Congestion forecast indicators with constant-pf and $-\mathrm{V}$ modes for scenario B 
is mainly due to consideration of $P-V$ nodes as explained in Section III-A2, where the node voltage remains fixed at specified values with reactive power compensation. Due to small CP for voltage deviation, there are no critical nodes identified in constant- $\mathrm{V}$ mode. However, it can be seen from Fig 4(b), that CP for branch currents to be more than $100 \%$ has slightly increased from 0.38 to 0.40 . Also, from Fig 4(c), the CP for transformer MVA loading to be more than $100 \%$ has increased approximately from 0.28 to 0.36 .

Thus, it is evident from the results that in constant- $\mathrm{V}$ mode, the CP for nodes voltage deviation congestion index has decreased, branch overloading has increased approximately from $38 \%$ to $42 \%$, transformer overloading from $28 \%$ to $36 \%$. The reason for the decreased voltage deviation is due to consideration of $P-V$ nodes. In constant- $\mathrm{V}$ mode, voltage is maintained at a specified value through reactive power compensation by injection of higher reactive current, which leads to higher current and MVA loading in associated branches and transformers.

Similar results can be obtained for several scenarios which could be used by the DSOs for visualizing the network congestion. These congestion indicators have potential to present a clear picture of network congestion before the system operator and ultimately help the DSOs in scheduling of their generation and demand resources present in the network.

\section{Conclusions}

This paper presents a congestion forecast framework which can be used to visualize the probability for the network congestion and voltage deviation in future distribution networks with large amount of solar PVs and PEVs. The core part of the framework is probabilistic power flow model which takes into account uncertainties associated with PVs production, load demand and PEVs charging. To demonstrate the use of the developed congestion forecast framework, case studies have been carried out using the framework to evaluate the impacts of PVs production level, load level, and PEVs, as well as the operating modes of PVs inverters on the network congestion. The results from the case studies highlight the facts that network congestion in branches and transformers is $30 \%$ and $20 \%$ respectively, more likely to occur when the level of local generations, demand and PEVs demand to increase by $100 \%, 95 \%$ and $100 \%$ respectively. Similarly, network congestion in branches and transformers is $4 \%$ and $8 \%$ respectively, more likely to occur in the constant- $\mathrm{V}$ mode as compared to constant-pf mode with the same generation and demand level. This correlation for a general case can be evaluated further using the approach but will be subject to future studies. The framework could be used by the DSOs to support them with the daily planning and management of the grids by efficiently scheduling local resources for both active and reactive power.

\section{REFERENCES}

[1] International Energy Agency, "Photovoltaic power systems programme annual report 2017," May 2018.
[2] J. Widén, M. Shepero, and J. Munkhammar, "Probabilistic load flow for power grids with high PV penetrations using copula-based modeling of spatially correlated solar irradiance," IEEE Journal of Photovoltaics, vol. 7, no. 6, pp. 1740-1745, 2017.

[3] M. E. Nassar and M. M. A. Salama, "A novel probabilistic load model and probabilistic power flow," in Proc. 2015 IEEE 28th Canadian Conference on Electrical and Computer Engineering, pp. 881-886.

[4] M. Fan and L. Huang, "Probabilistic power reserve evaluation algorithm for power systems considering high solar energy penetration," in Proc. 2018 IEEE Power and Energy Society General Meeting, pp. 1-5.

[5] Z. Ren, W. Yan, X. Zhao, Y. Li, and Y. Juan, "Probabilistic power flow for distribution networks with photovoltaic generators," in Proc. 2013 IEEE Power and Energy Society General Meeting, pp. 1-5.

[6] R. R. Appino, T. Mühlpfordt, T. Faulwasser, and V. Hagenmeyer, "On solving probabilistic load flow for radial grids using polynomial chaos," in Proc. 2017 IEEE PowerTech Manchester, pp. 1-6.

[7] G. Li and X. P. Zhang, "Modeling of plug-in hybrid electric vehicle charging demand in probabilistic power flow calculations," IEEE Transactions on Smart Grid, vol. 3, no. 1, pp. 492-499, 2012.

[8] N. G. Bhat, B. R. Prusty, and D. Jena, "Modeling of power demands of electric vehicles in correlated probabilistic load flow studies," in Proc. 2016 IEEE International Conference on Power Electronics, Drives and Energy Systems, pp. 1-6.

[9] S. Kong, H. C. Cho, J. U. Lee, and S. K. Joo, "Probabilistic modeling of electric vehicle charging load for probabilistic load flow," in Proc. 2012 IEEE Vehicle Power and Propulsion Conference, pp. 1010-1013.

[10] C. Wu, F. Wen, Y. Lou, and F. Xin, "Probabilistic load flow analysis of photovoltaic generation system with plug-in electric vehicles," International Journal of Electrical Power \& Energy Systems, vol. 64, pp. 1221-1228, 2015.

[11] A. G. Anastasiadis, E. Voreadi, and N. D. Hatziargyriou, "Probabilistic load flow methods with high integration of renewable energy sources and electric vehicles-case study of greece," in Proc. 2011 IEEE PowerTech Trondheim, pp. 1-8.

[12] A. Soroudi, M. Aien, and M. Ehsan, "A probabilistic modeling of photo voltaic modules and wind power generation impact on distribution networks," IEEE Systems Journal, vol. 6, no. 2, pp. 254-259, 2012.

[13] T. M. Masaud, R. D. Mistry, and P. K. Sen, "Placement of largescale utility-owned wind distributed generation based on probabilistic forecasting of line congestion," IET Renewable Power Generation, vol. 11, no. 7, pp. 979-986, 2017.

[14] C. Wan, J. Zhao, Y. Song, Z. Xu, J. Lin, and Z. Hu, "Photovoltaic and solar power forecasting for smart grid energy management," CSEE Journal of Power and Energy Systems, vol. 1, no. 4, pp. 38-46, 2015.

[15] IEEE 1547 Standard for Interconnecting Distributed Resources with Electric Power Systems, IEEE Std. 1547-2003, Jun. 2003.

[16] IEEE Standard for Interconnection and Interoperability of Distributed Energy Resources with Associated Electric Power Systems Interfaces, IEEE Std. 1547-2018, Feb. 2018.

[17] Nyregistreringar, "Bilsweden.se," [Online] Available: http://www.bilsweden.se/statistik/nyregistreringar [Accessed 18 Nov. 2018].

[18] C. S. Cheng and D. Shirmohammadi, "A three-phase power flow method for real-time distribution system analysis," IEEE Transactions on Power Systems, vol. 10, no. 2, pp. 671-679, 1995.

[19] M. Göransson and N. Larsson, "Development of an energy management model for Chalmers microgrid: Application for cost-benefit analysis of battery energy storage." Master's thesis, Chalmers University of Technology, Göteborg, Sweden, 2016.

[20] FED, "Fossil free energy districts," [Online] Available: https://www.uiainitiative.eu/en/uia-cities/gothenburg [Accessed 18 Nov. 2018]. 